\title{
Pigeons' FI behavior following signaled reinforcement duration
}

\author{
LYNN HOWERTON and DONALD MELTZER \\ Southern Illinois University, Carbondale, Illinois 62901
}

\begin{abstract}
Six pigeons were shifted to a two-component (constant and variable reinforcement magnitude) multiple schedule following fixed-interval (FI) training to a constant magnitude. In the variable component, cues associated with the smaller of two reinforcement levels eventually controlled longer pausing and lower overall response rates. In the constant component, five of six subjects' performance evidenced direct relationships between preceding reinforcement and pausing that appeared early and persisted through training. Largest differences in response distribution in the constant component occurred primarily in the middle portions of the FI. It was concluded that pause differences due to preceding reinforcement magnitude persist for a sustained period in a setting in which cued magnitude is contrasted in close temporal proximity.
\end{abstract}

The effects of preceding magnitude on fixed-interval (FI) behavior have been supported by many studies in the literature (e.g., Lowe, Davey, \& Harzem, 1974; Meltzer \& Howerton, 1973; Staddon, 1970). However, some studies have reported only transient effects of preceding magnitude (Harzem, Lowe, \& Davey, 1975) or differences due to preceding magnitude appearing only under special conditions (Jensen \& Fallon, 1973; Lowe, Harzem, \& Spencer, 1979).

Harzem et al. (1975) contend that context of reinforcement is important in producing magnitude effects on pausing. For example, Harzem et al. (1975) reported only temporary preceding magnitude effects on pausing in rats in a low-context setting in which three levels of sucrose concentration were varied over blocks of sessions. The pause difference was weak and transient. Similar weak differences in pausing associated with magnitude were reported by Lowe et al. (1979). Using pigeons, Lowe et al. (1979) reported pause differences occurring only with extreme levels (8 sec vs. $48 \mathrm{sec}$ ) of reinforcement duration, and then only at a long interreinforcement interval ( $\mathrm{FI}=8 \mathrm{~min}$ ).

The "low-context" designs and corresponding evidence of weak magnitude effects of Harzem et al. (1975) and Lowe et al. (1979) can be compared with the support for strong magnitude effects in the "high-context" designs of Meltzer and Howerton (1973), Staddon (1970), and others. For example, preceding magnitude effects were clearly demonstrated when FIs ended in

This research was conducted as part of a doctoral dissertation titled "Effects of Preceding Reinforcement on FI Performance" submitted to the Department of Psychology, Southern Illinois University at Carbondale. Some of the data were presented at the 1981 meeting of the Southwestern Psychological Association. I thank Donald Meltzer for his guidance and chairing the dissertation committee. Reprints may be obtained from Lynn Howerton, Department of Counselor Education and Psychology, Arkansas State University, State University, Arkansas 72467. different magnitudes that occurred in close temporal proximity within the same session whether unpredictable (Staddon, 1970) or with .50 probability in one component of a multiple schedule (Meltzer \& Howerton, 1975 ) or cycling strings of magnitude levels (Lowe et al., 1974).

The two studies that used pigeons as subjects represent extremes of the context continuum, with Staddon (1970) varying unpredictable noncued intervals within a session and Lowe et al. (1979) varying magnitude over sessions. The present study is designed such that magnitude varies within a session (high-context design), but two levels of predictable magnitude occur in one component of a multiple FI/FI schedule and alternate with a component containing constant magnitude. Consequently, predictable preceding magnitude effects can be observed in the constant-magnitude component.

\section{METHOD}

\section{Subjects}

Six mature pigeons were individually housed and maintained at $85 \%-90 \%$ of their free-feeding weights. Water was available at all times in the home cages. All subjects were experimentally naive.

\section{Apparatus}

The sound-attenuated experimental chamber had interior dimensions of $35 \times 47 \times 38 \mathrm{~cm}$ and was ventilated with a blower. The key required $.15 \mathrm{~N}$ to operate and was mounted behind a centered hole, $3.2 \mathrm{~cm}$ in diameter, punched in the front wall $26 \mathrm{~cm}$ above the floor. A Kodak Ectagraphic (Model AF) Carousel slide projector was mounted behind the key and projected either a diffuse amber light or white light with a $.6 \times 2.5 \mathrm{~cm}$ vertical or horizontal black bar. The reinforcer was access time to granulated Purina pigeon pellets through a $5 \times 6 \mathrm{~cm}$ rectangular opening presented by a solenoid-operated magazine. Reinforcement duration began when the bird's head broke a photocell light source inside the feeder, and it terminated according to the programmed reinforcement duration. A magazine light was the only source of illumination for the duration of food presentation. Otherwise, the key light and a diffused houselight were the sources of illumination. White noise produced by a Grason- 
Stadler noise generator (Model 901A) served as a masking source when presented to the chamber via 3.2-ohm speakers. Programming and recording equipment were located in the same room.

\section{Procedure}

Each subject was first allowed to eat food from the magazine then shaped to peck an amber key on a continuous reinforcement basis for 5 -sec access to chow. The time between responsecontingent food presentations was gradually increased until an FI 90-sec reinforcement schedule was reached. Subjects received 52 sessions on FI $90 \mathrm{sec}$, with each session consisting of 33 intervals. Approximately 30 sessions of magnitude discrimination training followed. The amber light continued to be correlated with 5-sec reinforcement, and these intervals constituted the constant-magnitude component of the multiple schedule and consisted of the 17 odd-numbered intervals. The remaining 16 even-numbered intervals formed the variable-magnitude component. Variable-magnitude component intervals ended with either $1-\mathrm{sec}$ or 9 -sec reinforcement duration with either a horizontal or a vertical bar correlated with each reinforcement level. Assignment of magnitude-discriminative stimuli was counterbalanced across subjects. Each magnitude level in the variable component occurred equally often and unpredictably within each session and changed daily to one of six counterbalanced orders.

\section{RESULTS}

Each subject acquired FI patterned behavior that stabilized during the last five sessions before magnitude discrimination. Each subject emitted approximately $10 \%$ of the responses in the first half of the interval and $30 \%-50 \%$ of the responses in the last sixth of the interval. Cumulative records revealed fairly stable responding and were characteristic of a break-and-run response pattern.

\section{Variable-Magnitude Component}

Over the course of the first five sessions (early in training), overall response rate was consistently higher to the 9-sec stimulus for S1 and S5 and was less consistent or showed no trend for the remaining four subjects. All subjects showed clear overall rate differences over the last five sessions (late in training). Considering the runrate measure, only $\mathrm{S} 1$ revealed a trend, with a higher run rate to the 9-sec cue in both early and late phases of training. The $1-\mathrm{sec}$ stimulus eventually controlled longer pausing than the 9-sec stimulus in all subjects. Initially, pause differences correlated with the two magnitude cues were mixed, but clear differences emerged in S1, S2, and S4 and moderate differences in S3, S5, and S6. The pause measures were converted to a relative pause measure and are pictorially presented in the left-hand portion of each subject's graph in Figure 1. For example, the proportional pause index for the 1-sec cue condition was computed for each session of a subject by. dividing the pause duration in the presence of the 1-sec stimulus by the total time pausing in all four stimulus conditions. So proportional pausing in the $1-\mathrm{sec}$ and 9-sec stimulus conditions in the variable component and the 1-sec and 9-sec (preceding magnitude) conditions in

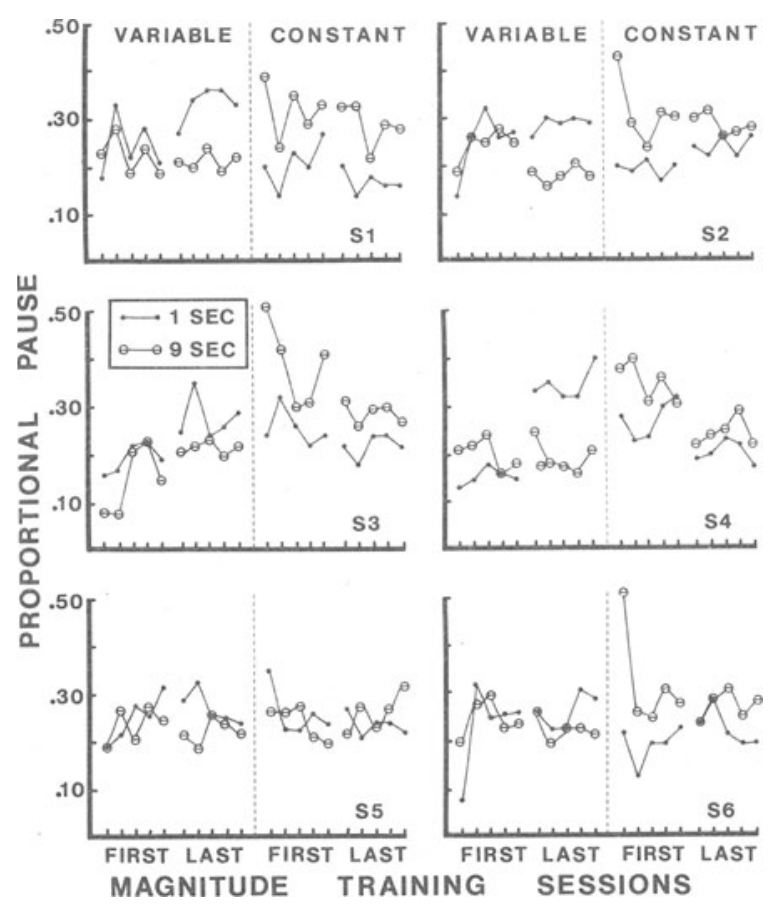

Figure 1. Proportional pause duration in the first five and last five sessions of magnitude training. The left-hand portion of each pigeon's graph is relative pausing to the discriminative cues signaling the 1- or 9-sec reinforcement duration. The right-hand portion of each graph is relative pausing following a 1- or 9-sec reinforcement duration of the previous interval during the discriminative cue for the 5 -sec reinforcement duration.

the constant component for each session of each subject will sum to 1.00 . As displayed in Figure 1, stimulus control of pausing developed later in training, with the 1-sec stimulus controlling proportionately more pausing than the 9-sec stimulus.

\section{Constant-Magnitude Component}

Early in training, four subjects' overall rates were higher after 1-sec reinforcement, S3 showed a trend in the opposite direction, and S5 showed no consistent effect. Overall response-rate differences following 1 -sec and 9-sec reinforcement tended to decrease with additional training. Table 1 shows mean run rate in the constant component for first and last five sessions of magnitude training. With the exception of one reversal for S6 early in training, run rates were higher following 1-sec reinforcement than following 9-sec reinforcement and persisted over training. With the pause measure, five subjects demonstrated longer pausing after 9-sec reinforcement than after 1 -sec reinforcement; this difference occurred early in training and persisted, although it lessened somewhat over sessions. The pause changes in S5 were inconsistent.

The right-hand portion of each subject's graph in Figure 1 displays the proportional pausing in the con- 
Table 1

Individual Subject Performance in the Constant Component With a Cue Associated With 5-Sec Reinforcement Duration But Preceded With Either a 1-Sec or 9-Sec Reinforcement Duration

\begin{tabular}{cccccc}
\hline & \multicolumn{3}{c}{ Mean Run Rate in the Constant Component } \\
\cline { 2 - 3 } Subject & \multicolumn{2}{c}{ First Five Sessions } & & \multicolumn{2}{c}{ Last Five Sessions } \\
\cline { 2 - 3 } \cline { 5 - 6 } & $1 \mathrm{Sec}$ & $9 \mathrm{Sec}$ & & $1 \mathrm{Sec}$ & $9 \mathrm{Sec}$ \\
\hline 1 & .74 & .65 & 1.00 & .90 \\
2 & 1.10 & .80 & 1.00 & .83 \\
3 & 1.36 & 1.21 & 1.50 & 1.34 \\
4 & 1.40 & 1.29 & 1.64 & 1.59 \\
5 & 1.47 & 1.23 & 1.72 & 1.65 \\
6 & 1.48 & 1.61 & 1.31 & 1.16 \\
\hline
\end{tabular}

Note-Run-rate measures (in responses per second) are means for the first and last five sessions of magnitude training for each preceding magnitude condition.

stant component. Comparing relative pausing, the birds showed pause differences in the constant component very early as pause differences developed in the variable component. S5 showed a weak relationship between the pause measure and preceding magnitude.

\section{Rate Changes Over Constant-Component Intervals}

Each subject increased its responding over the course of the interval. If terminal response rates in the constant component were analyzed over successive fifths of the interval, higher response rate followed 1-sec vs. 9-sec reinforcement, with substantially more difference in the middle portions of the interval. Subjects emitted proportionately, as well as absolutely, more responses in the middle of the interval following 1-sec magnitude.

\section{DISCUSSION}

The data are consistent with previous findings in the research literature. In the present study, overall rate varied inversely with preceding magnitude and pause duration appeared early and varied directly with preceding magnitude: both findings that are in agreement with pigeon and rat studies (Harzem et al., 1975; Lowe et al., 1974, Experiment 1; Staddon, 1970). Pause-duration changes appear to be robust with regard to the form of testing, and they appear in both rats and pigeons. The present study extends the generality of early onset of preceding magnitude effects on pausing using discriminative stimuli in a high-context setting.

The present study most closely approximates the design used by Meltzer and Howerton (1973, Experiment 1), although the latter used rats. Constant-component results were comparable, with more responses emitted after the lesser preceding magnitude in both studies and with the greater effects in the middle portions of the interval and a flatter distribution following the lesser magnitude. While other studies suggest differences between pigeon and rat FI performance (Lowe et al., 1979; Lowe \& Harzem, 1977), the influence of magnitude upon responses distributed throughout the interval in the present study are sirnilar to Meltzer and Howerton's (1973, Experiment 1) rat data.

According to Lowe and Harzem (1977), variables that increase the duration of the postreinforcement pause in the FI performance of rats and pigeons should increase the run rate in rats but not pigeons. However, Staddon (1970) reported that preceding magnitude had an inverse effect on run rate in pigeons, whereas Lowe et al. (1974, Experiment 1) reported the reverse, in that preceding magnitude had a direct effect on run rate in rats. The present run-rate data are consistent with Staddon's (1970) pigeon data. Apparently, Lowe and Harzem's (1977) contention is limited to pause-duration and run-rate changes at a particular level of an experimental manipulation (as in their study) but does not extend to comparisons across different levels (e.g., reinforcement magnitude), since pigeons show run-rate changes opposite to those of rats when preceding magnitude is varied.

The present study does not isolate why run rate varied inversely with preceding magnitude, since preceding magnitude and pause were confounded. Inherent in a high-context design, local behavioral contrast may operate such that preceding response rate may enhance pause duration in the next FI. However, no support for local behavioral contrast was observed in the present study.

\section{REFERENCES}

Harzem, P., Lowe, C. F., \& Davey, G. C. L. After-effects of reinforcement magnitude: Dependence upon context. Quarterly Journal of Experimental Psychology, 1975, 7, 579-584.

Jensen, C., \& FAllon, D. Behavioral after-effects of reinforcement and its omission as a function of reinforcement magnitude. Journal of the Experimental Analysis of Behavior, 1973, 19, 459-468.

Lowe, C. F., Davey, G. C. L., \& Harzem, P. Effects of reinforcement magnitude on interval and ratio schedules. Journal of the Experimental Analysis of Behavior, 1974, 22, 553-560.

Lowe, C. F., \& Harzem, P. Species differences in temporal control of behavior. Journal of the Experimental Analysis of Behavior, 1977, 28, 189-201.

Lowe, C. F., Harzem, P., \& Spencer, P. T. Temporal control of behavior and the power law. Journal of the Experimental Analysis of Behavior, 1979, 31, 333-343.

Meltzer, D., \& Howerton, D. L. Sequential effects of reinforcement magnitude on fixed-interval performance. Journal of Comparative and Physiological Psychology, 1973, 85, 361-366.

Meltzer, D., \& Howerton, D. L. Sequential effects of signaled and unsignaled variations in reinforcement magnitude on fixed interval performance. Bulletin of the Psychonomic Society, $1975,6,461-464$.

Staddon, J. E. R. Effect of reinforcement duration on fixedinterval responding. Journal of the Experimental Analysis of Behavior, 1970, 13, 9-11.

(Received for publication January 5, 1983.) 